\section{Dosisdichte Therapie verlängert krankheitsfreies Überleben bei Mammakarzinom}

\author{
In der adjuvanten Therapie des Mammakarzinoms gibt es eine Reihe \\ wichtiger Standardregimes. Jedoch ist der Stellenwert von 5-Fluorouracil \\ (5-FU) im FAC(5-FU/Doxorubicin/Cyclophosphamid)- bzw. FEC(5-FU/Epirubi- \\ cin/Cyclophosphamid)-Regime nicht ausreichend definiert, ebenso wenig \\ der Nutzen einer dosisdichten Gabe.
}

n der 4-armigen, randomisierten Phase-III-Studie wurden 2.091 Patientinnen mit nodal positivem, operablem Mammakarzinom behandelt. Sie erhielten adjuvant entweder das Standardregime EC-P (4 Zyklen Epirubicin 90 mg/ $\mathrm{m}^{2}$ plus Cyclophosphamid $600 \mathrm{mg} / \mathrm{m}^{2}$ an Tag 1, gefolgt von 4 Zyklen Paclitaxel $175 \mathrm{mg} / \mathrm{m}^{2}$ an Tag 1) alle 3 Wochen (q3w) oder dosisdicht alle 2 Wochen (q2w) mit Pegfilgrastim-Support. In 2 weiteren Armen wurde zu beiden Regimes zusätzlich 5-FU $\left(600 \mathrm{mg} / \mathrm{m}^{2}\right.$ an Tag 1) verabreicht.

Etwa die Hälfte der Patientinnen war postmenopausal, gut $90 \%$ hatten einen T1- oder T2-Tumor, knapp zwei Drittel
1-3 befallene Lymphknoten, HER2 war bei etwa einem Viertel überexprimiert und gut drei Viertel hatten einen positiven Hormonrezeptorstatus (Östrogenund/oder Progesteronrezeptor-positiv).

Unter dosisdichter Therapie stieg die krankheitsfreie Überlebensrate (DFS) nach 5 Jahren im Vergleich zum Standardintervall von 76 auf $81 \%$ (Hazard Ratio [HR] 0,77; p = 0,004). Die Rate für das 5-Jahres-Gesamtüberleben (OS) erhöhte sich von 89 auf $94 \%$ (HR 0,65; $\mathrm{p}=0,001$ ).

Die Zugabe von 5-FU zu EC-P führte dagegen nicht $\mathrm{zu}$ besseren Ergebnissen: Sowohl die 5-Jahres-DFS-Rate als auch die 5-Jahres-OS-Rate lagen im gleichen
Bereich (78 vs. $79 \%$ bzw. 91 vs. $92 \%$ ). Allerdings führte die Verdichtung der Dosis häufiger zu einer Grad-3/4-Anämie, $\mathrm{zu}$ einem Anstieg der Transaminasen und zu Myalgien. Die Anzahl der Grad-3/4-Neutropenien wurde dank der Gabe von Pegfilgrastim signifikant gesenkt (14,9 vs. 44,0\%). Unter der Zugabe von 5-FU stieg die Zahl der Neutropenien im Vergleich zu EC-P alleine von 24,2 auf $34,5 \%$ an.

Fazit: In der adjuvanten Therapie des frühen, nodal positiven Mammakarzinoms mit EC-P bewirkte die Verkürzung des Intervalls von 3 auf 2 Wochen (dosisdichtes Protokoll) unter Pegfilgrastim-Schutz eine Verbesserung des krankheitsfreien Überlebens. Die zusätzliche Gabe von 5-FU zu EC-P führte dagegen nicht zu einem Anstieg des krankheitsfreien Überlebens.

Brigitte Schalhorn

Del Mastro L et al. Fluorouracil and dose-dense chemotherapy in adjuvant treatment of patients with early-stage breast cancer: an openlabel, $2 \times 2$ factorial, randomised phase 3 trial. Lancet. 2015;385(9980):1863-72.

\title{
Operables Mammakarzinom: 12-Jahres-Daten zur adjuvanten Therapie
}

\begin{abstract}
Taxan-Typ und Applikationsschema schienen den Erfolg einer adjuvanten Therapie von Patientinnen mit einem operablen Mammakarzinom entscheidend zu beeinflussen. Jetzt liegen dazu Langzeitdaten vor.
\end{abstract}

\footnotetext{
n der ECOG-Studie E1199 wurde der Effekt der Taxane Paclitaxel und Docetaxel in 2 unterschiedlichen Behandlungsschemata (3-wöchigem oder 1-wöchigem Intervall) im Rahmen einer adjuvanten Brustkrebstherapie verglichen.
}

Insgesamt waren 4.954 Frauen mit einem Mammakarzinom im Stadium IIIII adjuvant behandelt worden: nach 4 Zyklen Doxorubicin plus Cyclophosphamid hatten sie in einem $2 \times 2$-faktoriellen Design randomisiert entweder Paclitaxel oder Docetaxel jeweils mit 4 Dosen q3w oder 12 Dosen q1w erhalten. Primä- rer Endpunkt der Studie war das progressionsfreie Überleben.

Die 5-Jahres-Ergebnisse zeigten, dass die sequenzielle Therapie mit 12 Zyklen Paclitaxel (q1w) nach 4 Zyklen Doxorubicin/Cyclophosphamid das krankheitsfreie, aber auch das Gesamtüberleben gegenüber der Paclitaxel-Applikation alle 3 Wochen signifikant verbesserte [Sparano JA et al. N Engl J Med. 2008; 358(16):1663-71].

Nun liegen die Langzeitdaten vor. Nach einem medianen Follow-up von 12,1 Jahren profitierten nur Patientinnen von einem signifikant günstigeren krankheitsfreien Intervall und einem längeren $\mathrm{Ge}$ samtüberleben, die Paclitaxel in 1-wöchigem Rhythmus (Hazard Ratio [HR] 0,84; $\mathrm{p}=0,011$ und HR 0,$87 ; \mathrm{p}=0,09)$ oder Docetaxel alle 3 Wochen (HR 0,78; $p=0,001$ und HR 0,86; $\mathrm{p}=0,054)$ erhalten hatten. Besonders Patientinnen mit triple-negativem Mammakarzinom profitierten von der dosisdichten Paclitaxel-Gabe. Dagegen führte die Dosisverdichtung bei Patientinnen mit hormonrezeptorpositivem oder HER2-negativem Tumor nicht zu einem besseren Gesamtüberleben. Auch zeigte sich, dass afroamerikanische Ethnie und Fettleibigkeit mit einem höheren Rezidiv- und Mortalitätsrisiko verbunden waren.

Fazit: Die in der ersten E1199-Auswertung beobachteten positiven Effekte einer wöchentlichen Paclitaxel-Therapie entsprachen qualitativ den Ergebnissen nach einem über 10-jährigen Follow-up. Explorativ analysierte Daten legen einen substanziellen Vorteil nur für Patientinnen mit einem triple-negativen Mammakarzinom nahe. Wolfgang Zimmermann

Sparano JA et al. Long-term follow-up of the E1199 Phase III trial evaluating the role of taxane and schedule in operable breast cancer. J Clin Oncol. 2015;33(21):2353-60. 